\title{
Phenotypic and morphometric characteristics of Bangkaew dog in Phitsanulok Province, Thailand
}

\author{
SUPHAWADEE YAEMKONG ${ }^{1, \vartheta}$, PRAPASIRI JAIPONG ${ }^{1, v »}$, PATTHANUN KOTHAM ${ }^{1, v \vee \downarrow}$, \\ TUAN NGUYEN NGOC ${ }^{1,2, \text {,rv }}$ \\ ${ }^{1}$ Program of Animal Science and Aquaculture, Faculty of Food and Agriculture Technology, Pibulsongkram Rajabhat University. 156, Plai Chumphon \\ Subdistrict, Mueang District, Phitsanulok 65000, Thailand. Tel./fax.: +66-55-267080, `email: suphayaku@ hotmail.com, vjprapasiri@hotmail.com, \\ vwp patthanun2309@gmail.com

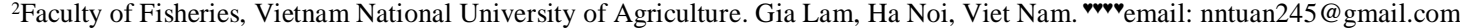

Manuscript received: 13 August 2019. Revision accepted: 7 September 2019.

\begin{abstract}
Yaemkong S, Jaipong P, Kotham P, Ngoc TN. 2019. Phenotypic and morphometric characteristics of Bangkaew dog in Phitsanulok Province, Thailand. Biodiversitas 20: 2877-2882. A research study was conducted to analyze phenotypic and morphometric characteristics of Bangkaew dogs in Mueang, Phromphirum, and Bangrakam districts, Phitsanulok Province, Thailand. In total, 54 Bangkaew dogs were randomly sampled by purposive sampling from 20 farmers between November 2018 and March 2019. The data consisted of nine phenotypic characteristics that were recorded visually and five morphometric characteristics that were obtained by using descriptive statistics and analysis of ANOVA to compare difference among means and correlation coefficients. Majority of the standard identities of Bangkaew dogs were according to the breed such as the guard coat around the face (100.00\%), the guard coat around the neck or the chest coat $(100.00 \%)$, the coat of belly $(100.00 \%)$, the guard coat behind ears $(100.00 \%)$, the lion's leg shape $(100.00 \%)$, the long coat from hip to the upper thigh $(100.00 \%)$, and the plume tail $(100.00 \%)$, except for the guard coat from the ruff around the shoulder $(98.15 \%)$. Moreover, the coat of hair of Bangkaew dogs in this study was white-brown color (55.31\%), followed by white-gray color $(23.92 \%)$, white-black color $(10.78 \%)$, brown color $(6.44 \%)$, gray color $(2.11 \%)$, and black color $(1.44 \%)$, respectively. Least square means of males morphometric characteristics was significantly $(\mathrm{p}<0.05)$ greater than female body weight $(21.44 \pm 3.33 \mathrm{~kg}$ vs. $19.04 \pm 3.01 \mathrm{~kg})$, body height $(49.52 \pm 4.09 \mathrm{~cm}$ vs. $46.21 \pm 5.30 \mathrm{~cm})$ and leg length $(38.64 \pm 8.88 \mathrm{~cm}$ vs. $34.31 \pm$ $5.75 \mathrm{~cm})$, except for body width $(19.48 \pm 5.82 \mathrm{~cm}$ vs. $17.76 \pm 4.24 \mathrm{~cm} ; \mathrm{p}>0.05)$, and for body length of females $(55.76 \pm 9.37 \mathrm{~cm}$ vs. $55.00 \pm 7.18 \mathrm{~cm} ; \mathrm{p}>0.05$ ) was superior to males of Bangkaew dogs. The correlations among morphometric characteristics such as body width vs. leg length, body length vs. body height, body weight vs. body height, body weight vs. body length, and body height vs. leg length, were $0.64,0.56,0.52,0.34$, and 0.33 , respectively. These results implied that in order to conserve standard identities and improve morphometric characteristics, their typical characteristics such as structure, coat of hair and body size required by raisers should be considered in the breeding selection and mating system.
\end{abstract}

Keywords: Bangkaew dogs, phenotypic and morphometric characteristics, Phitsanulok Province, Thailand

\section{INTRODUCTION}

The Bangkaew dog is a unique or signature indigenous breed of Phitsanulok Province, Thailand. This dog is a spitz-type working dog that originated in the Bangkaew, a small village located in the Bangrakam district, Phitsanulok Province in the Tha Nang Ngam area (Tangtrakanpong 1980; Yaemkong et al. 2018). Bangkaew Dog is an old breed that Thai people believe its ancestors descended from wild wolves. The breed traces its ancestry back to a cross between a native black and white female dog and a nowextinct wild dog. It is a medium-size, double-coated dog. The coats form the ruff around the neck and shoulders, forming a lion-like and a plumed tail, which is more noticeable on male than on female (Phavaphutanon and Laopiem 2011). The Bangkaew dog is an intelligent and dominant breed that is primarily known after its incredible loyalty and protective nature. This dog is brave, fearless, hardy, resourceful, clever, determined and more than eager to please his master, and not an aggressive breed but can be aloof with strangers and is very territorial and protective.
However, this breed is more likely to threaten than bite and attack. It makes a great guardian and watchdog that will loudly announce the arrival of any visitor (friend or stranger). There is a breed that is known to be very aggressive towards other dogs. The dog will get along well with other animals if they are raised together from a very young age. This breed is definitely for an experienced owner (Tagun 2000; Angkura et al. 2013). Nowadays, the rearing of Bangkaew dog is widely distributed in all country.

There are several studies on Bangkaew dogs rearing on farm, such as Amuneysit et al. (1993; 1994; 1995), Tagun (2000), Yaemkong et al. (2018), and Innak et al. (2019). However, a few studies were done for phenotypic and morphometric characteristics of Bangkaew dogs (Tangtrakanpong 1980; Amuneysit et al. 1992; Tagun 2000). In addition, a correlation between morphometric characteristics has not been described in the previous studies. Thus, the objective of this research was to investigate phenotypic and morphometric characteristics of Bangkaew dogs of small scale farmers in Phitsanulok 
Province, Thailand. The results from this study would provide important data and helpful information which can be used for conservation, breeding selection and mating system or improvement of Bangkaew dogs as well as helping farmers to avoid problem with their farms.

\section{MATERIALS AND METHODS}

\section{Description of study location}

The study was carried out in Mueang, Phromphirum, and Bangrakam districts, Phitsanulok Province, Thailand (Figure 1). Data collection was carried out between November 2018 and March 2019. Additional information on the area, topography, and climate (temperature, rainfall, and humidity) in Phitsanulok Province, Thailand can be found in Yaemkong and Tuan (2019).

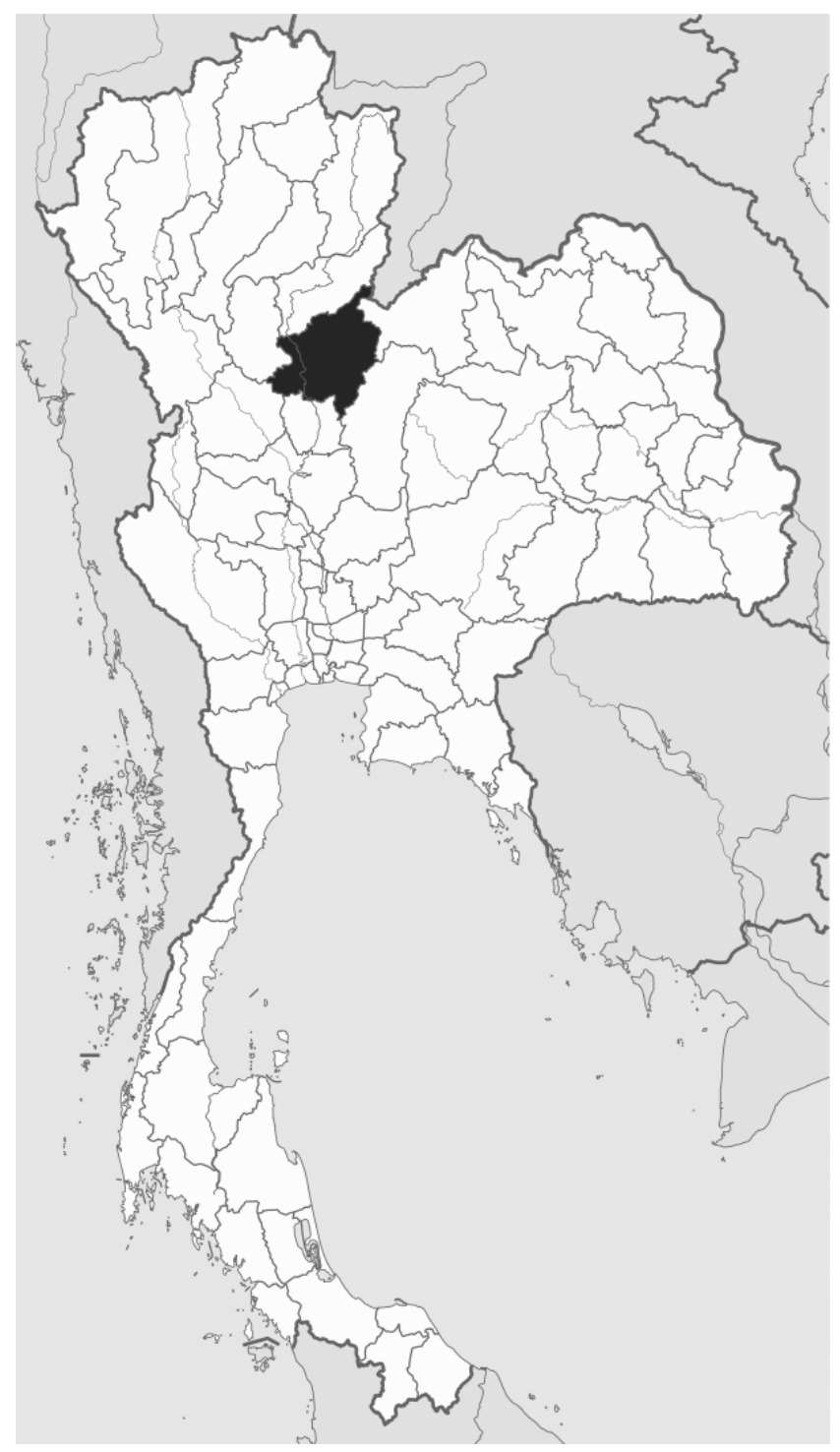

Figure 1. Study area in Phitsanulok Province, Thailand (Source: adapted from Wikipedia 2019)

\section{Sampling of animal and farm}

In total, 54 Bangkaew dogs, comprising of 25 mature males and 29 females, were randomly sampled by purposive sampling from 20 farmers in the study area. Dog raising farms in the region had been described in a previous study by Yaemkong et al. (2018). In this area, all of the Bangkaew dogs got vaccines such as rabies or hydrophobia, canine distemper, bronchitis, and leptospirosis. Most of the farmers in this study used family members to raise dogs. Feed was mixed by farmers or used commercial feed. Dogs were raised in either semi-freerange or in the cage rearing system. The majority of farmers reared Bangkaew dogs for selling as cash generation whereas the rest is keeping dogs as pets or for the contest.

\section{Characteristics and data preparation}

A total of nine phenotypic and five morphometric characteristics of both male and female Bangkaew dogs were analyzed in this study. Phenotypic characteristics were recorded visually following standard identities of Bangkaew dogs from Thai Bangkaew Kennel Club (TBK), Thailand. They consisted of guard coat around the face (GF), guard coat around the neck or the chest coat (CC), guard coat from the ruff around the shoulders (GS), coat of belly (CB), guard coat behind ears (GE), lion's leg shape (LL), long coat from hip to upper thigh (CHT), and plume tail (PL). Additionally, one basic characteristic was the coat of hair $(\mathrm{CH}$; white-brown, white-gray, white-black, brown, gray, and black color). Whereas, morphometric characteristics were recorded as follows: body weight (BW; $\mathrm{kg})$; body length $(\mathrm{BL} ; \mathrm{cm})$ : from the point of proserum or breastbone to the rear of the pelvis; body width $(\mathrm{BWi} ; \mathrm{cm})$, also called as chest circumference (the chest perimeter behind the forelegs); body height $(\mathrm{BH} ; \mathrm{cm})$ which was average between height at withers and hind height; and leg length (SL; cm): from lower belly to the toe. Weight and length in the study were measured by using an electrical balance and a ruler with $10 \mathrm{~g}$ and $1 \mathrm{~mm}$ sensitive scale respectively. The experiment procedure followed the protocols approved by the Ethical Committee of the Pibulsongkram Rajabhat University Animal Ethics Committee (Approval number: PSRU-AG-2018-004) Phitsanulok Province, Thailand.

\section{Analysis of data}

Data on phenotypic and morphometric characteristics were tabulated in terms of frequency and percentage. The morphometric characteristics were analyzed by the analysis of variance (ANOVA) differences were compared among means and correlation coefficients traits using the Proc Corr procedure of Statistical Analysis System (SAS 2004).

\section{RESULTS AND DISCUSSION}

\section{Phenotypic characteristics}

Basic phenotypic characteristics of Bangkaew dogs in Phitsanulok Province, Thailand in this study revealed that the primary of Bangkaew dogs (Figure 2). All of the 
Bangkaew dogs (both males and females) showed the guard coat around the face $(100 \%)$, the guard coat around the neck or the chest coat $(100 \%)$, the coat of belly $(100 \%)$, the guard coat behind ears $(100 \%)$, the lion's leg shape $(100 \%)$, the long coat from hip to upper thigh (100\%), and the plume tail $(100 \%)$ concerning with standard identities of Bangkaew dogs. However, only $98.15 \%$ of them had a guard coat from the ruff around the shoulder. The current study was in line with Angkura et al. (2013) who found that the majority of dogs had the characteristics of lips, nose color and nose muzzle, mouth shape, teeth, neck, waist, underline, front leg joints, and coat of hair in accordance with the breed standards while back feet, front feet, and back were not strongly related to the breed of dog. For comparison of characteristics against the breed identity, it was found that all of the $21 \mathrm{dogs}$ had shins in the lion's leg shape which were in accordance with the breed identity; while the coat of belly, the guard coat around neck, and the chest coat seemed not to follow any breed. Furthermore, Amuneysit et al. (1992) compared basic standard of Bangkaew dogs from four areas (Mueang, Wangthong and Bangrakam districts, and Chumsangsongkram sub-district) to The Preservation and Development of Thai Dogs of Bangkaew Breed in Thailand Association (PDBT) found that almost all standard characteristics of Bangkaew dogs, such as ear $(60.16 \%)$, double-coated $(65.04 \%)$, forearm $(70.73 \%)$, feet $(73.17 \%)$ and head $(78.86 \%)$ were not affected by breed identity. In addition, Tagun (2000) studied on morphological and karyotypic of Bangkaew dogs and related species in order to investigate the origin of the species, found that the morphological characteristics of Bangkaew dogs were similar to those of Asiatic Jackal, Asian wild dogs, and local Thai dogs. They have comparable hair and tail styles to Asiatic Jackal, the same shape of skulls and hair color as Asian wild dogs, and alike curly tails as local Thai dogs.

The coat of hair of Bangkaew dogs in Pitsanulok province, Thailand was found to have predominantly white-brown color $(55.31 \%)$, followed by white-gray color $(23.92 \%)$, white-black color $(10.78 \%)$, brown color $(6.44 \%)$, gray color $(2.11 \%)$, and black color $(1.44 \%)$, respectively (Figure 3 ). The results was in agreement with that reported by Amuneysit et al. (1992) who found that most of the coat hair had white-brown color $(48.78 \%)$, followed by white-black color $(22.76 \%)$, white-gray color (20.32\%), gray color (4.07\%), and black color (4.07\%), respectively. Moreover, Tagun (2000) reported that coat of hair from male and female Bangkaew dogs had whitebrown color (19.97\% and $64.67 \%)$, followed by white-gray color $(3.24 \%$ and $5.29 \%)$, white-black color $(2.22 \%$ and $4.10 \%)$, brown color $(0.17 \%$ and $0 \%)$, and gray color $(0 \%$ and $0.34 \%$ ), respectively. Additionally, Phavaphutanon and Laopiem (2011) confirmed that the color was usually white with shades of red, gray, brown, and black in a wide variety of Bangkaew dog patterns.

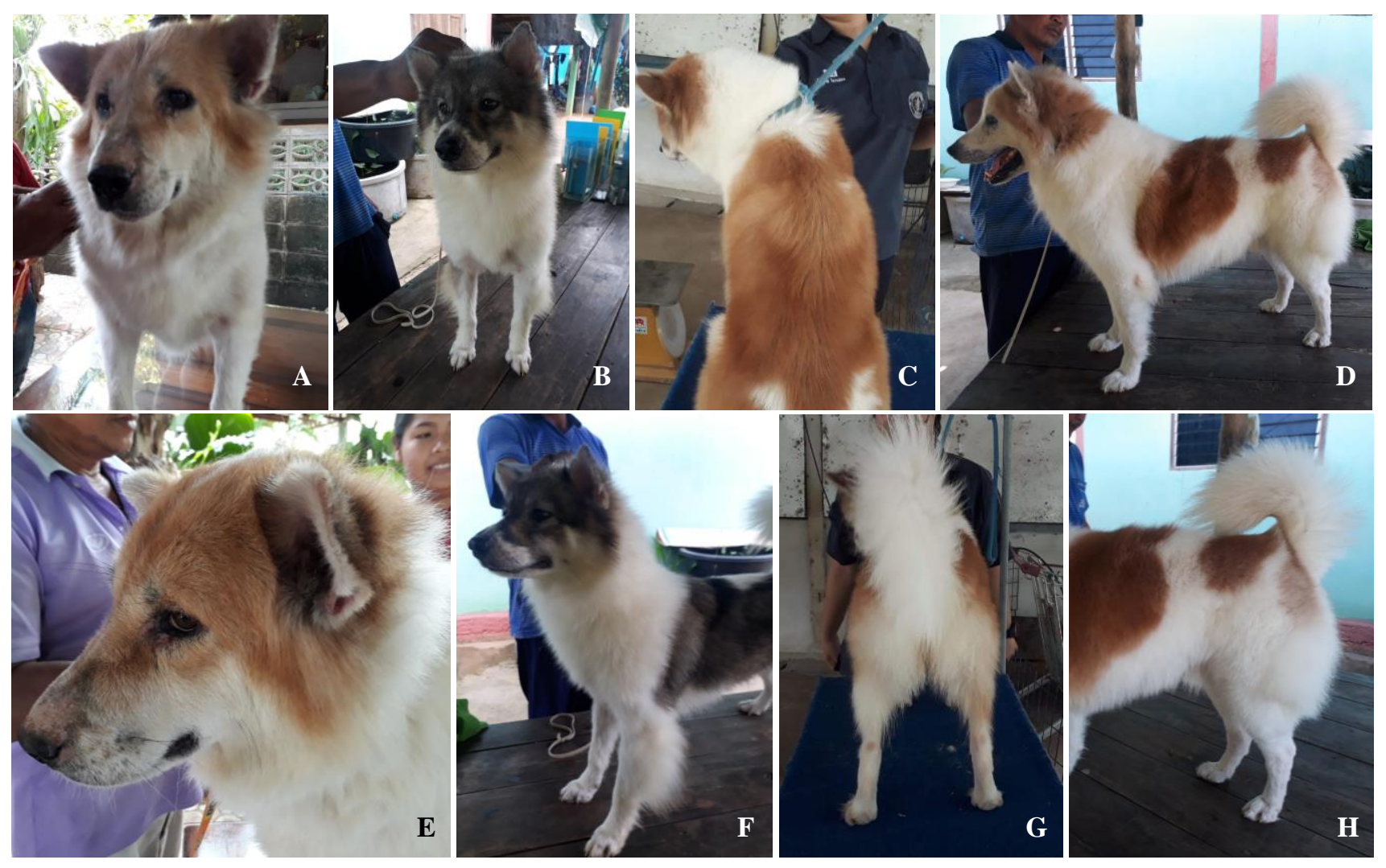

Figure 2. The Bangkaew dog phenotypic characteristics of standard identities (A) guard coat around the face (B) guard coat around the neck or the chest coat (C) guard coat from the ruff around the shoulders (D) coat of belly (E) guard coat behind ears (F) lion's leg shape $(\mathrm{G})$ long coat from hip to upper thigh and $(\mathrm{H})$ plume tail 

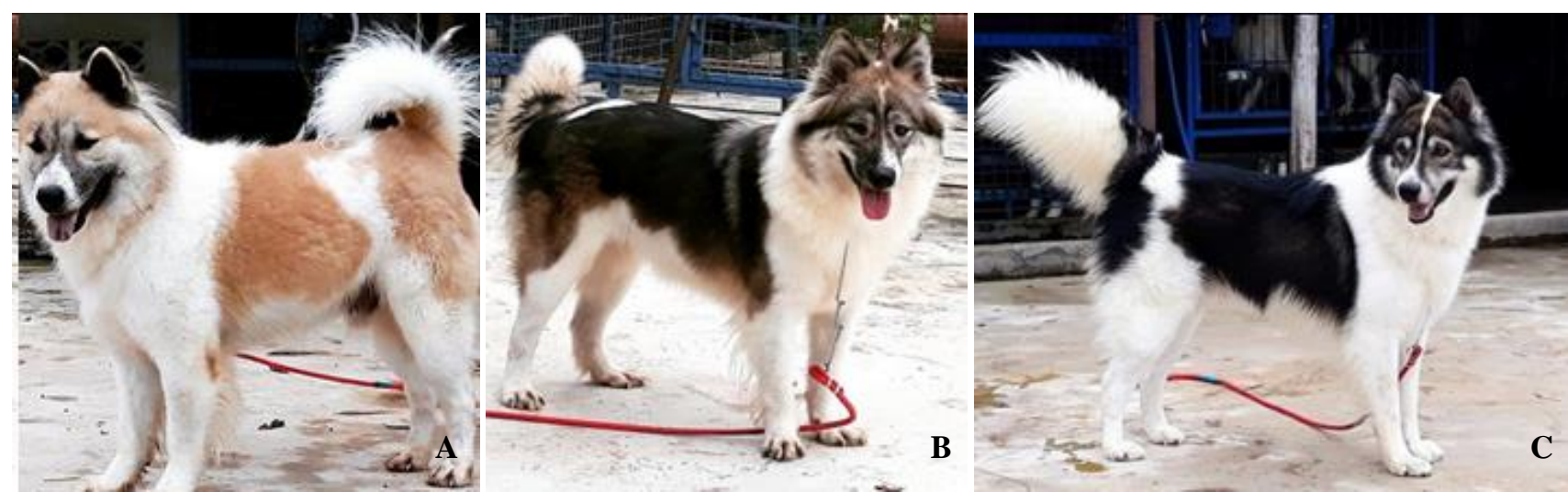

Figure 3. The phenotypic characteristics of coat of hair color (A) white-brown, (B) white-gray and (C) white-black

\section{Morphometric characteristics}

The least-square means of morphometric characteristics of Bangkaew dogs are presented in Table 1. The average values of morphometric characteristics from Bangkaew dogs in this study were closed to quantitative traits derived from related published papers of Thai Bangkaew dogs from Thailand (Amuneysit et al. 1992; Tagun 2000) and other dog breeds (Ibrahim 2008).

In this study, the male Bangkaew dogs showed greater performance traits than the female body weight, body height, leg length $(\mathrm{p}<0.05)$ and body width $(\mathrm{p}>0.05)$, except for body length $(\mathrm{p}>0.05)$. These values are similar to the study of Amuneysit et al. (1992) who reported that Thai Bangkaew dogs in Phitsanulok Province, Thailand had body weight ranged from 19 to $25 \mathrm{~kg}$ for male and 18 to $23 \mathrm{~kg}$ for female. Furthermore, Tagun (2000) reported that Bangkaew dogs had an average weight of $22 \mathrm{~kg}$ and 19 $\mathrm{kg}$, respectively, for male and female. A similar study was done by Ammar (2008) for Kababeesh dogs from Northwest of Sudan, this dog weighted from $21.42 \pm 0.88$ $\mathrm{kg}$ for male and $20.75 \pm 0.72 \mathrm{~kg}$ for female ones. According to the American Kennel Club (2000), those dogs were ranged medium size. However, they would be lighter than the Labrador retriever and the German Sheppard dog (Helmink 2000). Ammar (2008) reported that the body weight of dogs may vary from $1 \mathrm{~kg}$ in Chihuahua to $115 \mathrm{~kg}$ in St. Bernard. The time taken for growing a puppy to reach adult size will be longer for the large breeds as compared to the smaller ones.

The body height of dogs in this present study was similar to those values reported by Amuneysit et al. (1992). Bangkaew dogs in that study ranged from $48-53 \mathrm{~cm}$ for males and $43-48 \mathrm{~cm}$ for females. In the study carried out by Tagun (2000), the average height of dogs was $51 \mathrm{~cm}$ for male and $45 \mathrm{~cm}$ for female. In general, hindquarter of males was higher than that of females in both types. The height at the hindquarter in the Kababeesh type of females and males was found to be $85 \%$ and $92 \%$ of the height at withers, respectively. The Blue Nile type was found to be $98.5 \%$ and $100 \%$ for females and males. These estimations showed that the hindquarter of the Blue Nile type seemed to be equal or higher than the forequarters, especially in males as compared to those of the Kababeesh type. These implied that the growth rate of the forequarters started decreasing and tapered off earlier than the hindquarter in the Blue Nile type (Ammar, 2008).

The results showed that the body length and body width found to be in the same range for both genders and no significant differences were observed between females and males (Table 1). Even though there is no statistical difference, the value of body width of males is lengthier than females. Likewise, Ammar (2008) illustrated that males had a wider chest than females, although body length for female trended seemed to increase more than that of male. In the same way, Ammar (2008) showed that females demonstrated more stable body length growth than males from the Blue Nile.

Table 1. Least Square Means (LSM) and standard errors for morphometric characteristics of Bangkaew dogs in Phitsanulok Province, Thailand

\begin{tabular}{lccc}
\hline $\begin{array}{l}\text { Morphometric } \\
\text { characteristics }\end{array}$ & $\begin{array}{c}\text { Least Square } \\
\text { Means } \pm \\
\text { standard errors }\end{array}$ & SEM & P-Value \\
\hline Body weight $(\mathrm{kg})$ & $\begin{array}{c}20.17 \pm 3.36 \\
21.44 \pm 3.33^{\mathrm{a}}\end{array}$ & 0.67 & 0.0080 \\
Male & $19.04 \pm 3.01^{\mathrm{b}}$ & 0.57 & \\
Female & $55.41 \pm 8.36$ & & 0.7429 \\
& $55.00 \pm 7.18$ & 1.44 & \\
Body length (cm) & $55.76 \pm 9.37$ & 1.74 & \\
Male & & & 0.2155 \\
Female & $18.56 \pm 5.06$ & & \\
& $19.48 \pm 5.82$ & 1.16 & \\
Body width (cm) & $17.76 \pm 4.24$ & 0.79 & \\
Male & & & \\
Female & $47.74 \pm 5.02$ & & \\
& $49.52 \pm 4.09^{\mathrm{a}}$ & 0.82 & \\
Body height (cm) & $46.21 \pm 5.30^{\mathrm{b}}$ & 0.98 & \\
Male & & & \\
Female & $36.31 \pm 7.61$ & & \\
& $38.64 \pm 8.88^{\mathrm{a}}$ & 1.78 & \\
Leg length (cm) & $34.31 \pm 5.75^{\mathrm{b}}$ & 1.07 & \\
Male & & & \\
Female & &
\end{tabular}


Table 3. Correlation among morphometric characteristics of Bangkaew dogs in Phitsanulok Province, Thailand

\begin{tabular}{lcc}
\hline $\begin{array}{l}\text { Morphometric } \\
\text { characteristics }\end{array}$ & Correlation & P-Value \\
\hline Body weight & 0.34 & $0.0115^{*}$ \\
vs. Body length & 0.12 & 0.4019 \\
vs. Body width & 0.52 & $<0.0001^{* *}$ \\
vs. Body height & 0.09 & 0.5153 \\
vs. Leg length & & \\
& & \\
Body length & -0.01 & 0.9688 \\
vs. Body width & 0.56 & 0.4999 \\
vs. Body height & 0.09 & \\
vs. Leg length & & 0.0769 \\
& & $<0.0001^{* *}$ \\
Body width & 0.24 & \\
vs. Body height & 0.64 & $0.0157^{*}$ \\
vs. Leg length & & \\
Body height & & \\
vs. Leg length & 0.33 & and $0.01 \%$, \\
\hline $\begin{array}{l}\text { Note: } * * * \text { Correlation is significant at level } 0.05 \% \text { and } \\
\text { respectively }\end{array}$ & &
\end{tabular}

\section{Correlation among morphometric characteristics}

A half of positive relationships among the morphometric characteristics were significant $(\mathrm{p}<0.01$ and $\mathrm{p}<0.05$ ), except between BW and BWi, BW and SL, BL and $\mathrm{BWi}, \mathrm{BL}$ and $\mathrm{SL}$, and $\mathrm{BWi}$ and $\mathrm{BH}(\mathrm{p}>0.05$; Table 3$)$. The results found that the highest correlation is between BWi and SL (0.64), followed by correlation between BL and. $\mathrm{BH}(0.56)$, between $\mathrm{BW}$ and $\mathrm{BH}(0.52)$, between $\mathrm{BW}$ and $\mathrm{BL}(0.34)$, and the lowest correlation is between $\mathrm{BH}$ and SL (0.33).

However, the guidelines for the conservation and development of Bangkaew dogs were followed under standard identities breed. The development process should start with the study of details concerning the Bangkaew dog such as the physical structure, strong and weak points, pedigree history, characteristics of pedigree transmission, genetics and breeding by the selection of sire and dam which can increase the strong points and also decrease the weak points. Especially, the breeders should pay more attention to the pedigree history data recording system. They should carefully and correctly record details of the dog in accordance with the facts (Phavaphutanon and Laopiem 2011; Marie-Dominique and Savolainen 2013).

The study indicated that most phenotypic characteristics of Bangkaew dogs were along with standard identities breed (the guard coat around the face, the guard coat around the neck or the chest coat, the coat of belly, the guard coat behind ears, the lion's leg shape, the long coat from hip to upper thigh, and the plume tail while the main coat of hair was white-brown, followed by white-gray, white-black, brown, gray, and black, respectively. The male Bangkaew dogs in almost each morphometrical characteristics showed a higher value than those of females $(p<0.05)$, except for body length $(p>0.05)$. Significant positive correlations were between body width and leg length, body length and body height, body weight and body height, body weight and body length, and body height and leg length, respectively. These findings suggested that the farmers in the region should follow the guidelines for the development of Thai Bangkaew dogs and cooperate together in the selection of distinguished breeds for further development.

\section{ACKNOWLEDGEMENTS}

This study was undertaken with financial support from the Pibulsongkram Rajabhat University, Thailand. The authors would like to thank Bangkaew dog farmers, staff, and students for participation during the study.

\section{REFERENCES}

American Kennel Club. 2000. AKC Board Minutes website. www.akc.org/breeds/german_shepherd_dog/

Ammar MEI. 2008. Phenotypic characteristics and genetic diversity of Sudanese indigenous Canines. [Dissertation]. University of Khartoum, Sudan.

Amuneysit P, Navanukraha B, Chalearmsan N, Sriwattanasombat P, Sribuntao S. 1992. The investigation of basic data in characteristics of Thai Bangkwar's dog: 1) Characteristic of hair and phenotype. In: Proceedings of the $31^{\text {st }}$ Kasetsart University Annual Conference: Animal Science, Fisheries and Veterinary Science, Bangkok, 22-25 January 1992. [Thailand]

Amuneysit P, Navanukraha B, Chalearmsan N, Sriwattanasombat P, Sribuntao S. 1993. The investigation of basic data in characteristics of Thai Bangkwar's dog, Phitsanulok Province: 2) Management. In: $9^{\text {th }}$ proceedings of Academic Rajamangala Institute of Technology: Agriculture. Lampang, 20-22 January 1993. [Thailand]

Amuneysit P, Navanukraha B, Chalearmsan N, Sriwattanasombat P, Sribuntao S. 1994. Notice from breeding of Thai Bangkwar's dog, Phitsanulok Province. In: $10^{\text {th }}$ proceedings of Academic Rajamangala Institute of Technology: Agriculture. Bangkok, 22-24 January 1994. [Thailand]

Amuneysit P, Navanukraha B, Chalearmsan N, Sriwattanasombat P, Sribuntao S. 1995. A study of feeding by company on production performance of Thai Bangkwar's dog: 1) Blood parameter. In: $11^{\text {th }}$ proceedings of Academic Rajamangala Institute of Technology: Agriculture. Bangkok, 22-24 January 1995. [Thailand]

Angkura R, Karndee P, Changkasemsuk T. 2013. Development Guideline for Thai Spitz: Thai Bang Kaew Dog to Be a New Internationally Recognized Breed of Dog: A Social Science Dimension Study [Dissertation]. Sukhothai Thammathirat Open University, Bangkok. [Thailand]

Helmink SK. 2000. Analysis of weight and height in a dog guide population to optimize mature size. [Dissertation]. University of Illinois at Urbana Champaign, Illinois.

Ibrahim AME. 2008. Phenotypic Characteristics and Genetic Diversity of Sudanese indigenous Canines. [Dissertation]. Department of Genetics and Animal Breeding, Faculty of Animal Production, University of Khartoum, Sudan.

Innak N, Wannaporn N, Jaipong P, Yaemkong S, Nguyen Ngoc T, Khwanchanok N, Kotham P. 2019. Situation and local wisdom for Thai bangkaew dog farm in Phitsanulok Province. Khon Kaen Agr J 47(Suppl. 2): 1009-1016. [Thailand]

Phavaphutanon J, Laopiem S. 2011. Evaluation of Microsatellite Polymorphism and Genetic. Variability in Thai Ridgeback and Bangkaew Dogs. Thai J Vet Med. 41(3): 273-282.

SAS. 2004. SAS 9.13 Help and documentation. SAS Institute Inc., Cary, NC, USA

Tagun J. 2000. Morphology and karyotypes of Bangkaew dogs and related species. [Dissertation]. Chulalongkorn University, Bangkok. [Thailand]

Tangtrakanpong N. 1980. Bangkwar's dog. Trakun Thai Printing, Phitsanulok. [Thailand] 
Wikipedia. 2019. Map of Thailand highlighting Phitsanulok Province https://en.wikipedia.org/wiki/Phitsanulok_Province\#/media/File:Thail and_Phitsanulok_locator_map.svg

Yaemkong S, Sea-ui S, Thippayaporn A, Nguyen Ngoc T, Gothom P, Jaipong P, Yaemkong S. 2018. A Study on Situation of Bang Kaew Dog Rearing of Farmers in Mueang and BangRakam Districts, Phitsanulok Province. In: The $5^{\text {th }}$ Rajabhat University National \&
International Research and Academic Conference, Phetchaburi, Thailand, 3-5 December 2018.

Yaemkong S, Tuan NN. 2019. Diversity of phenotypic characteristics of White Tailed-Yellow Chicken populations reared under free-range system in Phitsanulok Province, Thailand. Biodiversitas 20 (5): 12711280 . 